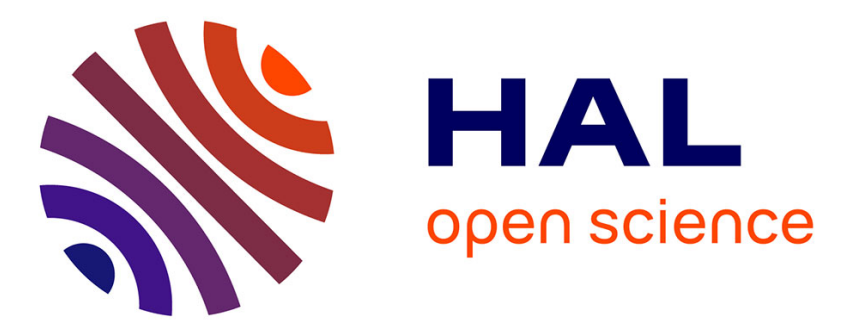

\title{
Summary of 21st joint EU-US transport task force workshop (Leysin, September 5-8, 2016)
}

P. Mantica, C. Bourdelle, Y. Camenen, R. Dejarnac, T. Evans, T. Goerler, J. Hillesheim, Y. Idomura, M. Jakubowski, P. Ricci, et al.

\section{- To cite this version:}

P. Mantica, C. Bourdelle, Y. Camenen, R. Dejarnac, T. Evans, et al.. Summary of 21st joint EU-US transport task force workshop (Leysin, September 5-8, 2016). Nuclear Fusion, 2017, 57 (8), pp.087001. 10.1088/1741-4326/aa753f . hal-01785563

\section{HAL Id: hal-01785563 https://hal.science/hal-01785563}

Submitted on 15 Jul 2021

HAL is a multi-disciplinary open access archive for the deposit and dissemination of scientific research documents, whether they are published or not. The documents may come from teaching and research institutions in France or abroad, or from public or private research centers.
L'archive ouverte pluridisciplinaire HAL, est destinée au dépôt et à la diffusion de documents scientifiques de niveau recherche, publiés ou non, émanant des établissements d'enseignement et de recherche français ou étrangers, des laboratoires publics ou privés. 


\title{
Summary of $21^{\text {st }}$ Joint EU-US Transport Task Force Workshop (Leysin, September 5-8, 2016)
}

P.Mantica $^{1}$, C.Bourdelle ${ }^{2}$, Y.Camenen ${ }^{3}$, R.Dejarnac ${ }^{4}$, T.Evans ${ }^{5}$, T.Görler ${ }^{6}$, J.Hillesheim ${ }^{7}$, Y.Idomura $^{8}$, M. Jakubowski ${ }^{9}$, P.Ricci ${ }^{10}$, A.White ${ }^{11}$ and the Participants to the $21^{\text {st }}$ Joint EUUS Transport Task Force Workshop*

${ }^{1}$ Istituto di Fisica del Plasma, CNR, Milano, Italy

${ }^{2}$ CEA, IRFM, F-13108 Saint Paul Lez Durance, France

${ }^{3}$ CNRS, Aix-Marseille Univ., PIIM UMR7345, Marseille, France

${ }^{4}$ Institute of Plasma Physics, Czech Academy of Sciences, Prague, Czech Republic

${ }^{5}$ General Atomics, P.O. Box 85608, San Diego, California

${ }^{6}$ Max Planck Institut für Plasmaphysik, Garching, Germany

${ }^{7}$ CCFE, Culham Science Centre, Abingdon, OX14 3DB, UK

${ }^{8}$ Japan Atomic Energy Agency, Tokyo 110-0015, Japan

${ }^{9}$ Max Planck Institut für Plasmaphysik, Greifswald, Germany

${ }^{10}$ Swiss Plasma Center, EPFL, Lausanne, Switzerland

${ }^{11}$ Plasma Science and Fusion Center, MIT, Cambridge, MA, USA

\begin{abstract}
This conference report summarizes the contributions to, and discussions at, the $21^{\text {st }}$ Joint EUUS Transport Task Force workshop, held in Leysin, Switzerland, during 5-8 September 2016. The workshop was organized under 8 topics: progress towards full-F kinetic turbulence simulation; high and low $\mathrm{Z}$ impurity transport, control and effects on plasma confinement; 3D effects on core and edge transport (including MHD, external fields and stellarators); predictive experimental design; electron heat transport and multi-scale integration; understanding power decay length in the SOL; role of the SOL in the L-H transition; validation of fundamental turbulence properties against turbulence measurements. This report follows the same structure.
\end{abstract}

\section{Introduction}

The $21^{\text {st }}$ Joint EU-US Transport Task Force workshop was held in Leysin, Switzerland, during 5-8 September 2016, with the Swiss Plasma Center (SPC) as host. The EU TTF Chair P.Mantica (IFP-CNR, IT) and Deputy Chair C.Bourdelle (CEA, FR) took care of the workshop organization with the help of P.Ricci (SPC, CH, Chair of the LOC) and the collaboration of the US TTF Chairs, J. Rice (MIT, MA) and G. Staebler (GA, CA), of the Asian Pacific TTF Chair, H.Jhang (NFRI, Rep. of Korea) and of the other members of the Programme Committee.

The workshop was organized with 8 plenary sessions including invited, oral and poster contributions on the 8 topics, and additional posters on the two general topics of core and pedestal transport. Each topical session was coordinated by one or two session chairs. The session topics were: progress towards full-F kinetic turbulence simulation (chair Y.Idomura, JAEA, Japan); high and low Z impurity transport, control and effects on plasma confinement (chair Y.Camenen, CNRS, FR); 3D effects on core and edge transport (including MHD, external fields and stellarators) (chairs M. Jakubowski, IPP Greifswald, D, T. Evans, GA, US); predictive experimental design (chair A. White, MIT, US); electron heat transport and multi-scale integration (chair T.Goerler, IPP Garching, D); understanding power decay length

\footnotetext{
${ }^{*}$ The list of all participants to the $21^{\text {st }}$ Joint EU-US TTF workshop can be found at http://spc.epfl.ch/ttf16
} 
in the SOL (chair P.Ricci, SPC, CH); role of the SOL in the L-H transition (chair R.Dejarnac, IPP Prague, CZ); validation of fundamental turbulence properties against turbulence measurements momentum transport (chair J-Hillesheim, CCFE, UK). The chairs were in charge of the scientific organization of their sessions, proposing to the whole Programme Committee the selection of invited and oral contributions for their sessions. They also chaired their respective sessions, led the discussions and provided written input to the present report.

The workshop was attended by 97 participants, of which 66 from EU, 18 from US, 13 from Asian countries. There were 25 invited talks, 18 contributed orals and 41 posters. This workshop was the first hold in Europe within the EUROfusion framework. The EUROfusion General Assembly has approved the continuation of the EU TTF as partner of the US and Asian TTFs to maintain the regular organization of the joint workshops, which provide useful feedback into the EUROfusion work-programme. Financial support from EUROfusion for participation to the meetings is limited to the Chairs. For the biennium 2017-2018 C.Bourdelle has taken up the role of EU TTF Chair and R. Mc Dermott was voted during the Leysin workshop as the new Deputy Chair. The wiki page of the EU TTF can be found at https://www2.euro-fusion.org/eu-ttf/.

In order to promote new ideas and a free discussion, it is traditional for TTF workshops not to publish full proceedings. Subject to author consensus, a large subset of the presentations was made available under password protection on the workshop web-site http://spc.epfl.ch/ttf16, where also all abstracts are publicly available. It was agreed at the workshop to prepare a short conference report for publication in a plasma physics journal, in order to provide a useful feedback to the whole fusion community and in line with previous TTF workshops. The scope of the present report is therefore to provide a concise summary of the results and issues discussed at the workshop. The content will be organized in 8 sections corresponding to the session topics listed above, with a final section summarizing progress achieved, open issues and directions for future work. The affiliations of the authors are not reported to avoid making the text too heavy, but they can all be found on the workshop website under the Abstracts section.

\section{Progress towards full-F kinetic turbulence simulation}

This session was newly proposed in the $21^{\text {st }}$ EU-US TTF Workshop. The session consisted of five talks (three invited and two oral) and two posters, and a discussion on the high priority issues in the development of full-f gyrokinetic codes.

In the first invited talk, G. Dif-Pradalier presented full-f gyrokinetic simulations on the self-organization of meso-scale $\mathrm{E} \times \mathrm{B}$ flows called the " $\mathrm{E} \times \mathrm{B}$ staircase" and the edge-core interplay of plasma turbulence. In this work, fixed-flux and fixed-gradient versions of the global full-f semi-Lagrangian code GYSELA were used. Firstly, physics properties of the $\mathrm{E} \times \mathrm{B}$ staircase in fixed-flux ion temperature gradient driven (ITG) turbulence simulations were discussed, and it was shown that this structure disappears in fixed-gradient simulations, where an adaptive source model affects the meso-scale structures, and that near critical transport with and without the $\mathrm{E} \times \mathrm{B}$ staircase are qualitatively and quantitatively different. Expected properties of $\mathrm{E} \times \mathrm{B}$ staircases were compared against fluctuation measurements using the fast-sweeping reflectometry on Tore Supra. It was shown that the step width of the $E \times B$ staircase is mesoscale $\sim 40 \rho_{\mathrm{i}}$, and that the location of its micro-barriers is weakly correlated to low order mode rational q surfaces, where $\rho_{\mathrm{i}}$ is the ion Larmor radius and $\mathrm{q}$ is the safety factor profile. It was also shown that in the experiment, the $\mathrm{E} \times \mathrm{B}$ staircase is observed mainly in the Saturated Ohmic Confinement (SOC) regime, where the ITG turbulence is considered to be dominant, while it disappears in the Linear Ohmic Confinement (LOC) regime, which is characterized by the trapped electron mode (TEM) turbulence. Secondly, radial profiles of density fluctuations were compared between GYSELA and Tore Supra, and a reasonable 
agreement was obtained. Comparisons between simulations with stable and unstable edge profiles showed that the density fluctuations in the marginal plasma core are significantly affected by the edge turbulence, suggesting important roles of the spreading of edge turbulence and the resulting edge-core interplay in global turbulence simulations.

In the second invited talk, S. Leerink presented full-f gyrokinetic simulations of the FT-2 tokamak using the global full-f particle code ELMFIRE, in which kinetic electrons are treated using an implicit approach. In this work, systematic validation studies against the confinement scaling in FT-2 tokamak were discussed. Although fluctuation spectra from the Doppler reflectometry and the frequency, correlation and propagation of the geodesic acoustic mode were successfully reproduced, systematic under prediction of transport levels occurred in the edge region. To resolve this issue, a limiter model was introduced, and the grid number was increased by developing a three dimensional (3D) domain decomposition method. In the validation study, a particular focus was set on the isotope effect. On FT-2, the particle confinement in deuterium plasmas is improved by $\sim 40 \%$ compared to hydrogen plasmas at similar plasma parameters. ELMFIRE reproduced this isotope effect, and it was found that the density fluctuation amplitudes are comparable or higher in deuterium plasmas, while the particle transport in deuterium plasmas is reduced by a favorable shift in the cross-phase between the density fluctuations and the radial turbulent convection.

In the third invited talk, C. S. Chang presented full-f gyrokinetic simulations of the edge turbulence using the global full-f particle code XGC1, in which various electron models from drift-kinetic electrons to fluid electrons are implemented. The talk focused on the heat-flux width on divertor plates in the attached plasma condition, and on trigger mechanisms of the $\mathrm{L}-\mathrm{H}$ transition. In the former part, it was shown that XGC1 reproduces the experimental scaling of the divertor heat-flux width observed in multiple tokamak devices, and its extrapolation to ITER was discussed. Although the scaling predicts an extremely narrow heatflux width, XGC1 predicted 5x wider heat-flux width, suggesting the possibility of attached plasma operation in ITER. In the second part, a new strategy to simulate L-H transition physics was discussed. Although the L-H transition develops in transport time scales, recent turbulence measurements on DIII-D demonstrate that the L-H transition is triggered by a rapidly increasing turbulence-driven shear flow that develops $\sim 100 \mu$ s prior to the L-H transition. This fast shear flow development was simulated using XGC1.

In the following oral session, $\mathrm{K}$. Imadera presented full-f gyrokinetic simulations of the ITG turbulence with momentum input using the global full-f Eulerian code GKNET. This work focused on the asymmetry of the ion temperature stiffness between co-current and counter-current neutral beam injection (NBI) in experiments. The simulation results indicated that the stiffness in the outside of the mid-minor radius becomes weaker with co-current momentum input, when it is imposed near the mid-minor radius. The mechanism was discussed based on the radial electric field determined by the radial force balance relation. The co-current rotation and the resulting radial electric field lead to the profile shear residual stress, which enhances the co-current rotation, while the counter-current rotation is weakened by the profile shear residual stress. S. Matsuoka presented full-f gyrokinetic simulations of neoclassical toroidal viscosity (NTV) induced by non-axisymmetric magnetic perturbations. From the NTV benchmark between the global full-f Eulerian code GT5D and the global delta$\mathrm{f}$ particle code FORTEC3D, it was shown that NTV in global kinetic calculations depend on the collisionality and its magnitude is significantly lower than that predicted in the so-called superbanana plateau theory, which predicts collisionality independent NTV.

In the poster session, J. Anderson presented a methodology to analyze non-Gaussian probability distribution functions (PDFs) of intermittent turbulent transport in global full-f gyrokinetic simulations. In this work, the Auto-Regressive Integrated Moving Average model is applied to time series data of intermittent turbulent heat transport to separate noise and 
oscillatory trends, and non-Gaussian features of the PDFs were extracted. It was then shown that non-Gaussian tails of the PDFs from first principles based gyrokinetic simulations agree with an analytical estimation based on a two fluid model. Y. Idomura presented full-f gyrokinetic simulations of electron heating modulation numerical experiments using GT5D, which uses a hybrid kinetic electron model. GT5D qualitatively reproduced the density peaking and rotation changes observed in electron cyclotron resonance heating (ECRH) modulation experiments on ASDEX-U. It was shown that electron heating induces the transition from the ITG turbulence to the TEM turbulence, which leads to the reversal of particle transport, and that the resulting particle pinch and the rotation changes in the countercurrent direction are closely linked through the toroidal canonical angular momentum conservation.

In the discussion session, the capability and limitation of current global full-f gyrokinetic simulations were discussed focusing mainly on treatments of kinetic electrons and electromagnetic perturbations. It was also pointed out that a close comparison against $1.5 \mathrm{D}$ transport codes with turbulent transport models is needed to demonstrate the utility of global full-f gyrokinetic simulations.

\section{High and low $Z$ impurity transport, control and effects on plasma confinement}

About half of the impurity session contributions were dedicated to impurity transport by neoclassical and turbulent processes and the other half to the impact impurities have on transport and confinement.

To start the session, T. Pütterich discussed the operating constraints that impurities put on a reactor. The burn window was assessed by balancing the fusion power and the power losses (transport and radiation) in a 0D model. Low $\mathrm{Z}$ impurities reduce the fusion power by dilution and high $\mathrm{Z}$ impurities enhance radiation losses. Increasing the concentration of low $\mathrm{Z}$ impurities quickly leads to the disappearance of the burn window, emphasizing the critical role of He transport in a reactor. Impurity limits prove to be even stricter when profile effects are taken into account with the plasma core highly vulnerable to radiation. This implies that hollow impurity profiles are desirable, for low and high $\mathrm{Z}$, and that high $\mathrm{Z}$ impurities are better mantle radiators in the sense that they radiate less at core temperature than at edge temperature. In the second introductory talk, R. Guirlet reviewed the different instrumental techniques used to characterize impurities in fusion plasmas, from the edge (infra-red and visible) to the core (soft-X). Emphasis was given to the interpretation of the data for transport studies, discussing measurements for ion populations that are not in equilibrium and the impact of line integration.

Concerning impurity transport, the difficulty faced by the theory to accurately predict the peaking of low to medium Z impurity (He to N) discussed at the TTF 2014 in Culham was further confirmed in recent modelling of JET ITER-like wall L-modes by N. Bonanomi. In these ICRH heated plasmas, the difference between the predicted and measured normalised impurity density gradient is found to be typically $\Delta \mathrm{R} / \mathrm{L}_{\mathrm{nZ}} \sim 1$ with a peaking that can be under or over predicted depending on the cases. The modelling includes neoclassical (local driftkinetic simulations with NEO) and turbulent transport (local quasi-linear and non-linear gyrokinetic simulations with GENE). To improve our prediction of the impurity peaking, the interplay between neoclassical and turbulent processes, that is usually neglected, has recently been explored. For $\rho_{*} \rightarrow 0$, the neoclassical and turbulent problems are decoupled and can be treated separately. At finite $\rho *$, this is no longer true and the two processes interact proportionally to $\rho *$. P. Manas focused on the impact of the neoclassical equilibrium on turbulent transport, i.e. how the deviation of the stationary solution from a Maxwellian modifies turbulent transport. The study was conducted with the $\delta$ f gyrokinetic code GKW and complemented with an analytical fluid model. The main outcome is that the neoclassical 
equilibrium gives rise to an additional contribution to roto-diffusion which increases with collisionality and changes sign with the direction of the plasma current. Y. Sarazin focused on the other side of this problem and showed that long-lived $\mathrm{m}=1$ structures generated by turbulence significantly affect neoclassical transport in global full-f gyrokinetic simulations (GYSELA). How relevant these two mechanisms are for the modelling of current experiments remains to be quantitatively assessed.

For high $\mathrm{Z}$ impurity transport, the situation is comparatively better and the experimental observations are reasonably well captured by the modelling provided the enhancement of the neoclassical pinch generated by poloidal asymmetries is taken into account. Modern neoclassical codes like NEO can be used to compute the neoclassical transport in the presence of poloidal asymmetries. Alternatively, analytical formulae have been derived in the limit of impurities in the Pfirsch- Schlüter regime, main ions in the banana regime and low impurity Mach number. In view of performing integrated modelling of tungsten transport, for which the full numerical solution is too costly, S. Breton tested the validity of the analytical formula for realistic JET parameters. The analytical solution was compared to NEO results showing that by adjusting the coefficient governing temperature screening the analytical solution can be applied beyond its theoretical limits. This provides a convenient tool for integrated modelling and the model is under implementation in JETTO. In the same vein, S. Newton presented an analytical derivation of the neoclassical impurity transport and bootstrap current in a stellarator valid for high $Z$ collisional impurities and bulk ions in the $1 / v$ regime. Interestingly, high $Z$ impurities are found to be affected by temperature screening, as in tokamaks. The analytical model is being compared to the drift-kinetic solver SFINCS.

On the experimental side, perturbative studies performed on RFX were presented by L. Carraro. In RFX plasmas, the main ion density profile is usually flat or slightly peaked and the impurity profile strongly hollow. Impurity injection by laser blow-off $(\mathrm{Ni})$, gas puff $(\mathrm{Ne})$ and pellets $(\mathrm{C}, \mathrm{Ne}, \mathrm{Li})$ showed that these hollow profiles result from a strong outward convection layer close to the edge, concurrent to a reduced diffusion in the same region. This is observed irrespectively of the injected impurity, with no obvious dependence on the impurity charge or on the confinement state (single or multi-helicity). The observed level of transport is much larger than classical collisional transport in a stochastic field. The possible role of micro-tearing turbulence is under investigation. The impact of sawteeth on impurity transport was addressed by R. McDermott in AUG. Charge exchange measurements of the He and B density were performed in AUG plasmas with a large $\mathrm{q}=1$ radius and long sawteeth ( $100 \mathrm{~ms}$ ). Conditional sampling was used to reconstruct the temporal evolution of the impurity density across a sawtooth cycle. The B profile is observed to be hollow and to flatten at the sawtooth crash. In contrast, the He profile is mostly flat and kicks up at the sawtooth crash. A simple full-reconnection model qualitatively reproduces the B density behaviour but not the He one, which remains a mystery.

Concerning the impact of impurities on transport, two theoretical studies focusing on the pedestal region were presented. The first one by I. Pusztai focused on neoclassical transport and the second one by D. Hatch on turbulent transport. These two studies were motivated by the strong and unexpected impact the ITER-like wall (ILW) had on the JET pedestal. I. Pusztai showed that the description of poloidal flows in the pedestal region requires a global treatment and that the local approach fails to capture their large inboard/outboard asymmetry. In the presence of non-trace impurities, neoclassical main ion and impurity fluxes are found in the same direction and the neoclassical momentum flux significantly increases with the effective charge. D. Hatch presented local and global gyrokinetic simulations (GENE) of the pedestal region of a JET H-mode. For the reference low $\mathrm{Z}_{\text {eff }}$ case, the experimental heat flux is quantitatively recovered by adding the contributions from neoclassical transport, ion scale turbulent transport and electron scale turbulent transport computed in three separate 
simulations. Ion scales were found to be dominated by micro-tearing modes (MTM) and electron scales by electron temperature gradient modes (ETG). Increasing the pedestal top temperature results in a strong increase of the heat flux, mostly due to the destabilisation of ITG and ETG modes. A reduced level of transport can be obtained at high temperature by increasing the low $\mathrm{Z}$ impurity content whose main effect is to reduce the ITG turbulence by main ion dilution. This mechanism is proposed to explain the major trends of the ILW operation.

Experiments on the impact of impurity injection on transport and confinement were performed on AUG with He, in C-Mod with Ne and on JET with He and N. On AUG and JET, A. Kappatou showed that increasing the He concentration up to $10 \%$ leads to a decrease of the stored energy by about $10 \%$ in AUG N-seeded $\mathrm{H}$-modes and by $25 \%$ in JET baseline scenario. In AUG half of the stored energy change is due to the main ion dilution. The remaining loss may be due to the reappearance of the high field side high density structure previously demonstrated to decrease confinement due to its impact on the alignement between the temperature and density pedestals. The He puff also results in the decrease of the $\mathrm{N}$ concentation in the core and in an increase in the ELM frequency. In JET the stored energy reduction is dominated by the impact of He on the NBI power deposition, which is found more outward with He injection due to the higher pedestal density. A higher ELM frequency is observed in the presence of He, as in AUG. When the ELM frequency is kept at its nohelium level by gas puffing, the pedestal density and the NBI deposition are not strongly affected by the He injection and a much smaller impact of He on confinement is observed. $\mathrm{M}$. Porkolab presented recent results on the impact of Ne injection in C-Mod Ohmic L-modes. As in AUG low confinement plasmas, no effect of the impurity injection on the global energy confinement time is observed. The ion temperature is found to be higher by about $10 \%$ in the seeded discharge, however, yielding an increased neutron rate. In JET ILW L-modes, N. Bonanomi showed that $\mathrm{N}$-seeded discharge $(\sim 1 \%)$ have higher ion and electron temperature. Linear and non-linear gyrokinetic simulations (GENE) show that the reduction in transport is a combination of the main ion dilution, the change in s/q due to the change in resistivity (higher Zeff ), the higher collisionality and the lower $T_{e} / T_{i}$. These three experimental studies seem to indicate that the impact of impurities on transport and confinement is rather complex, with several direct and indirect effects competing to determine the overall impact on the energy confinement time.

\section{3D effects on core and edge transport (including MHD, external fields and stellarators)}

Three-dimensional (3D) magnetic field perturbations have many interesting and possibly useful effects in magnetic fusion plasmas. During the session, devoted to the influence of 3D topological effects on the plasma core and edge, several aspects of such phenomena have been covered. In particular, the aim was to identify physics mechanisms that can be qualitatively or quantitatively linked to changes in the core and edge transport caused by applied or intrinsic 3D magnetic perturbation. This includes the effects of $\mathrm{E} \times \mathrm{B}$ convection caused by magnetic islands and/or stochasticity, the coupling of 3D fields to turbulent flows, effects on transport due to changes in rotation and magnetic perturbation effects on forward and backward spontaneous confinement transitions such as core and edge transport barriers.

A large part of the session was devoted to the understanding of transport in the core of the stellarator. A. Alonso gave an invited talk on impurity dynamics in the 3D topology of stellarators, which is also important for understanding their radial transport, where the parallel projection of the momentum conservation equation for an impurity species requires a balance among the forces that are responsible for the impurity radial flux. During the presentation, recent experimental observations in the TJ-II stellarator related to the transient impurity 
behaviour following the injection of hydrogen pellets during stationary neutral-beam-heated plasmas have been presented. Experimentally, this transient behavior is manifested as a low frequency oscillation in the radiation monitors (AXUV diodes). The working hypothesis is that this oscillation is due to the presence of a sizeable variation of the impurity density on flux surfaces, which is a result of the balance of several parallel forces acting on the impurities. This balance is broken by the fast changes in the plasma parameters induced by the pellet and relaxes into a new equilibrium displaying a damped oscillation in the process.

A fluid-dynamical model of the impurity species is used to confirm this interpretation and shed light on the physics of the oscillation frequency and lifetime. The model includes the parallel pressure gradient, friction with main ions and inertia in the parallel momentum conservation equation. This is combined with the particle conservation equation to give dynamical equations for the relative impurity density variation and parallel flow. Importantly, the model treats the real three-dimensional magnetic configuration of TJ-II and can be easily adapted to axisymmetric configurations. The model shows that the oscillation is due to the $\mathrm{E} \times \mathrm{B}$ advection of the $\mathrm{m}=1$ impurity density variation, whereas the damping is mediated through the parallel compression of the parallel impurity flow.

In a related contributed talk A. Mollén, et al. discussed the important role of the electric field on impurities in stellarator - in particular, due to flux-surface variations of the electrostatic potential. These variations are expected to have a small impact on the transport of the bulk plasma species, however earlier work has found that they can be significant for plasma impurities which are more prone to be affected because of their high charge.

The continuum code SFINCS and the particle-in-cell code EUTERPE were used to solve the radially local drift-kinetic equation in 3D geometry in order to calculate neoclassical particle fluxes, and account for flux-surface potential variations. In the presented work the results for trace impurity $\left(\mathrm{C}^{6+}\right)$ fluxes for a Large Helical Device (LHD) equilibrium have been presented. SFINCS and EUTERPE conform well with each other, both in terms of predicting the impurity fluxes and predicting the potential variations. The results indicate that even for a low- $Z$ impurity such as $\mathrm{C}^{6+}$ the transport can be substantially affected by the varying potential.

The part of the oral session devoted to 3D effects on transport in stellarator was complemented by a poster presentation by J. Proll on gyrokinetic turbulence in the initial operation of Wendelstein 7-X (W7-X). The newly built, optimised, W7-X stellarator in Greifswald promises strongly reduced neoclassical transport and is an ideal candidate to study turbulent transport. In her work, Proll gave examples on how the use of gyrokinetic simulations can guide the development of dedicated turbulence measurements. One dedicated experiment was suggested to test a theoretical model on quasi-isodynamicity and enhanced trapped-electron mode (TEM) stability, stating that quasi-isodynamic configurations (of which $\mathrm{W} 7-\mathrm{X}$ is an approximation) should be stable towards density-gradient-driven TEMs as long as the electron temperature gradient is not too large. This theory was shown to be valid in perfectly quasi-isodynamic configurations thanks to the absence of trapped particles whose processional drift frequency is resonant with the propagation of drift waves necessary to drive TEMs unstable. For the dedicated experiment, the standard configuration (SC) and the highmirror (HM) configuration of $\mathrm{W} 7-\mathrm{X}$ were compared. The SC is expected to have significantly more particles where the aforementioned resonance is present than the HM configuration. Just as expected, the linear growth rates measured in the simulations were higher in the SC than in the HM configuration. This should lead to an increase in turbulence, which, if indeed measured in the experiment, would confirm the theory. In addition, Proll, et al. suggested that a variation of the temperature ratio of electrons and ions should lead to a variation of the heat flux levels, if - as suspected - the heat flux was mainly caused by micro turbulence. 
Stability and confinement of tokamak H-mode operation depend crucially on properties of the plasma pedestal - the outer region of the plasmas characterized by steep pressure gradients and enhanced edge currents. Both of these effects lead to so-called Edge Localized Modes (ELMs) - frequent instabilities connected with sudden release of large fraction of plasma particles and energy towards the first wall. It is possible to eliminate ELMs with help of resonant magnetic perturbations (RMPs), which is also accompanied by changes in core and edge transport. As RMPs are envisaged for ITER the second part of the session was devoted to the influence of resonant magnetic perturbations on edge and core plasmas.

The effect of the RMP on the plasma equilibrium can be represented by a non-resonant part (ideal MHD kink response) and a resonant part (island formation at rational surfaces). D. Orlov, in an invited talk, discussed recent experiments from DIII-D in which H-mode discharges were exposed to periodic RMP $n=360 \mathrm{deg}$. toroidal phase flips. This showed clear differences in the transport during each RMP phase flip with an asymmetric change in particle confinement resulting in a recovery of the line average density to its pre-RMP value. At the same time, the pedestal density recovers somewhat but remains slightly below its pre-RMP value and ELMs continue to be suppressed. This transport asymmetry is hypothesized to be due to destructive or constructive coupling of the RMP field to the static intrinsic error fields in the plasma edge in the two toroidal RMP coil phases. These experiments have allowed to locate the position of the first plasma response to changes in the RMP field to a region near the $q=3$ surface that correlates with the region of vanishing electron perpendicular velocity within the uncertainties of the measurements. The plasma response, seen in both the density and toroidal momentum, propagates radially away from this surface reaching the core of the plasma faster than the edge.

Orlov also addressed an urgent ITER issue for demonstrating whether toroidallymonochromatic RMP spectrum is a necessary condition for the suppression of ELMs. This is required in case some of the ITER RMP coils malfunctions, e.g. due to neutron irradiation. Robust ELM suppression has been reproducibly obtained on DIII-D during experiments in which various non-axisymmetric coil loops were turned off. RMP ELM suppression was achieved with as few as 5 out of 12 I-coils on DIII-D at similar coil currents. The impurity transport in these discharges was found to be similar to that in the QH-mode and ELMing discharges with $40 \mathrm{~Hz}$ ELMs. It was also found that the ELMs suppression was accompanied by rapid increase in the plasma rotation in all cases indicating a significant reduction in toroidal momentum transport during suppression. Numerical modelling with the ideal equilibrium perturbation code IPEC, the linear resistive MHD stability code MARS and the resistive MHD code M3D-C1 all show a 50\% decrease in the dominant $n=3$ toroidal mode number perturbation followed by increased contribution in the $n=2$ side-bands. Results of the MAFOT separatrix manifold modelling code also show transition from 3 to 2 footprint lobes on the divertor. It was also shown that in this set of discharges different perturbation spectra had a surprisingly very small effect on the global transport and turbulence.

In the last part of the presentation by Orlov a set of experiments focused on understanding the influence of the resonant part of the magnetic perturbation on the edge topology and transport of inner-wall limited L-mode plasmas was discussed. A modulated ECH pulse technique was used in plasmas with a large $n=1, m=2$ island created by RMP fields to study the interaction of the ECH pulses with the islands. Periodic toroidal phase flips were used to study the transport in the island $\mathrm{O}$ - and $\mathrm{X}$-point regions. The results show spontaneous bifurcations in heat transport across the islands. Two hypotheses are being examined to account for this transport bifurcation. One is the spontaneous change of magnetic islands topology between the nested flux surfaces and stochastic states. The other is a transition in the turbulent transport inside the islands. The response to magnetic perturbations is by far more complex than a simple "density pump-out" mechanism, i.e. a rapid loss of electron density 
due to an increased electron mobility in the stochastic magnetic field. It is still an open issue what is the exact mechanism of the kinetic response to externally applied perturbations.

In this sense, as presented in the invited talk by G. Spizzo, early measurements with MPs during the 80 's, in the University of Texas tokamak TEXT, showed the appearance of macroscopic $\mathrm{E} \times \mathrm{B}$ convective cells associated with the MP. This result was confirmed by the measurement of a 2D map of floating potential, in a small Japanese tokamak, in the same year. These very early results have been recently revived, since measurements of plasma potential have been experimentally determined in great detail in the edge of the RFX-mod reversed-field pinch (RFP) and of the TEXTOR tokamak, with applied magnetic perturbations (MP's). Results presented by Spizzo show that a magnetic island in the plasma edge can indeed act as a convective cell, which, once formed, dominates the heat and particle transport in this region.

Generally speaking, in these new measurements the plasma potential has approximately the form $\Phi(r, \theta, \phi ; t)=\Phi_{0}(\mathrm{r}) \sin [u(\theta, \phi ; t)]$, with the helical angle defined as $u=m \theta-n \phi+$ $\omega t$. This implies a strong correlation between the magnetic field topology and the poloidal/toroidal modulation of the measured plasma potential. This helical modulation has been explained assuming that in a chaotic tokamak edge, the ion and electron drifts yield a predominantly electron driven radial diffusion when approaching the island X-point (which is a driver of chaos), while ion diffusivities are generally an order of magnitude smaller. In the O-point, on the contrary, closed flux surfaces are generally found, and ion and electron diffusivities are comparable. This results in a strong, positive radial electric field $E_{r}>0$ pointing outward in the island O-point, and a vanishing $E_{r} \approx 0$ right into the O-point. This mechanism has been often neglected on the grounds that electron drifts are small, but this is not the case in a chaotic environment. In RFX-mod the picture is more complicated, since Xpoints can act both as drivers of electron diffusion, or dynamical traps (reduced electron diffusion), depending on the helicity of the dominant island. In the latter case, the radial electric field structure points inward, to the island O-point. The assumption on electron/ion motion in the vicinity of magnetic islands has been verified in detail via the guiding-centre code ORBIT - an analytical model for the plasma potential is implemented in the guiding centre equations of motion, and analyses of the ambipolar flow show that both ion- and electron-dominated transport regimes can exist, known as ion and electron roots in stellarators. A good agreement is found between measured and modelled plasma potential, in the "electron root" case: in particular, ORBIT reproduces the vanishing $\mathrm{E}_{\mathrm{r}} \approx 0$ in the island $\mathrm{O}$ point and the positive $\mathrm{E}_{\mathrm{r}}>0$ near the X-point, in the TEXTOR case.

There are many experimental observations indicating that when RMPs are applied, the radial electric field in the edge plasma becomes less negative or even positive and the plasma is accelerated toroidally in the co-current direction. This does not always require a presence of an island. E. Kaveeva in her invited talk discussed other mechanisms, which lead to the change of the radial electric field during RMPs. The electron current is proportional to the difference between the electric field and the positive field, of the Boltzmann type, which should stop electrons. It should be compensated by the radial ion current, which arises if the poloidal rotation of the plasma differs from the neoclassical one. The balance of the currents determines the radial electric field and the poloidal rotation of the pedestal plasma. While the currents of ions and electrons have opposite directions, the flows of both species are directed outwards: electrons escape the pedestal mainly along the braided magnetic field lines while ions due to the neoclassical conductivity by means of the radial convective motion. The result is the decrease of the pedestal density, i.e. the pump-out effect. The theory was tested during modeling studies with B2SOLPS5.2 and SOLPS-ITER codes. The modeling shows that for ASDEX-Upgrade and MAST parameters the pump-out effect is significant and can explain a major part of the density drop in H-mode. 
For a wide range of plasma parameters the toroidal rotation can change significantly during the RMPs application. While the electrons escaping along the magnetic field lines produce no toroidal torque, the radial current of ions gives rise to the torque associated with the toroidal projection of $\vec{j} \times \vec{B}$ force. This force is co-current directed and it should be compensated by the radial transport of toroidal momentum due to anomalous radial viscosity. This effect is localized in the stochastic layer (unlike NTV) and leads to additional co-current rotation.

The plasma response to RMPs includes the screening of external magnetic perturbations. The current of escaping electrons having the significant radial component after averaging over the flux surface, locally has much bigger toroidal and poloidal components. The different Fourier harmonics of electron current are localized in the vicinity of corresponding rational flux surfaces and modify the harmonics of magnetic field perturbation. Generally, these currents lead to the screening of RMPs. The screening is absent for the harmonics for which the Boltzmann electric field for electrons coincides with neoclassical electric field at their rational flux surfaces. Two different regimes of screening are found: the 'ion' branch corresponding to the negative radial electric field, which is close to the neoclassical one, and the 'electron' branch for which the electric field is positive. The transition from ion to electron branch during the rise of RMPs occurs gradually for existing tokamaks and abruptly for ITER parameters, leading to the bifurcation in the RMPs penetration into the plasma.

For sufficiently large RMP fields, a region with open field lines is created. In tokamaks with a poloidal divertor, this causes the separatrix to split into a tangle of helical lobes, which guide open magnetic field lines onto the divertor targets. This opens an outward transport channel for particles and energy, resulting in a flow pattern that is determined by the position and poloidal mode number of the resonant magnetic surfaces The open field line region is also realized in stellarators, i.e. with an island divertor at $\mathrm{W} 7-\mathrm{X}$.

In his invited talk H. Frerichs presented the EMC3-EIRENE code package, which provides a 3D coupled transport solver for the fluid edge plasma in self-consistent interaction with neutral gas. This allows for a detailed analysis of the impact of RMPs in tokamaks and intrinsically non-axisymmetric stellarator configurations for which it has been originally targeted. Under high recycling conditions, a flow reversal in the regular scrape-off layer (SOL) alternates with downstream flow inside the separatrix lobes. While friction between counter flows has been identified as the mechanism for an early transition into detachment at W7-AS (predecessor of W7-X), the impact in tokamak RMP configurations is not yet understood.

The strength of the EMC3-EIRENE code is its flexible geometry module, which has allowed the exploration of advanced divertor configurations (e.g. for the TCV snowflake divertor and their interaction with RMPs). However, discrepancies between modelling results and experimental observations have been identified and explored for an RMP H-mode configuration at DIII-D. Often the vacuum RMP field is good enough to qualify the impact of RMPs, while a quantitative analysis requires the consideration of plasma response effects which may lead to partial screening or amplification of the applied RMP field. These have to be taken from external codes such as M3D-C1, and uncertainties remain due to assumptions in the MHD model therein (e.g. single fluid vs. two fluid, linear vs. non-linear). On the other hand, edge and divertor modelling requires plasma-neutral and plasma-surface interactions which are included in EMC3-EIRENE, and it has been found that discrepancies in the modelled and observed heat flux striation pattern can be related to recycling conditions.

\section{Predictive experimental design}

To improve predictive capability for transport is one major goal of the TTF, by synthesis of theory, modelling and experiments. Predictions are always made in the community using a variety of tools. Past observations: Plasma discharges can be very reproducible, predictions 
based on lots of experimental data tend to be pretty good. Extrapolation from data: 0-D scaling laws based on experiments used for machine design, e.g. ITER, DEMO, etc. Theory based models: represent major research thrusts and can be used to predict details of turbulence and transport. It is the latter that constituted the focus of the session on "Predictive experimental design", or "predict first" for short.

One may assume that a session on "experimental predictions" focuses on the use of integrated models to develop simulations of the time evolution of a tokamak discharge. In practice, scientists also rely on less integrated techniques, and extremely valuable, directly measureable results can still be obtained. This session (the first of its kind at TTF) is intended to highlight the use of theory-based predictions in order to optimize the preparation of an experiment or diagnostic design and set-up. Certainly integrated modeling was discussed by some session speakers (and by many others at the TTF), but the real purpose of the session was to provide examples of the use of stand-alone theory based codes (e.g. GYRO or GENE) to aid in the experiment preparation phase. The codes can be used effectively to determine whether a given physics question can be answered in a planned experiment, what kind of results would be expected from theory and what margins outside experimental uncertainties are available to discriminate a certain effect. Predictions using gyrokinetic simulations, together with both qualitative and quantitative comparisons to experiments, were discussed in detail. The session showcased predictions for changes both in turbulence and in transport. Through these talks and discussions, the TTF hopes to motivate more "predict first" efforts.

The first invited talk in the session, by P.Snyder, covered a range of predict-first experiments focusing on pedestal structure, with an emphasis on tests of the EPED model, and use of this model to predict a new regime, the Super H-Mode. The EPED model is a relatively simple predictive model for the pedestal structure, which employs two calculated constraints to predict the height and width of the pedestal. The model hypothesizes that, in "high performance" H-modes such as Type I ELM and Quiescent H-Mode, the pedestal is constrained by global peeling-ballooning (PB) modes, driven by pressure and current gradients across the edge barrier. In addition, while numerous mechanisms drive transport across the pedestal, it is hypothesized that the combination of strong $\mathrm{E} \times \mathrm{B}$ shear, together with heat and particle sources, cause the pressure gradient to generally continue to rise until it is constrained by the onset of kinetic ballooning modes (KBM). The EPED model takes as input a set of scalar inputs and uses these to construct series of model equilibria to calculate PB and pedestal-integrated KBM constraints, and combines these to predict the pedestal height and width. The model, because it requires only scalar inputs, is particularly amenable to use in predict-first experiments. Several predict-first tests of the model were described, beginning with dedicated experiments on DIII-D in 2008, which varied the plasma current, field and triangularity to vary the pedestal height more than an order of magnitude, finding agreement in the measured pedestal height and width to $\sim 20 \%$. Later predict-first experiments on Alcator C-Mod at high field led to the development of an improved treatment of diamagnetic stabilization of PB modes. A wide comparison, including dedicated rhostar scans, was later conducted, combining predict-first results with other comparisons on JET, AUG, JT-60U, DIII-D, C-Mod, and recently COMPASS. Similar levels of agreement ( $\sigma \sim 0.2-0.25$ with no free or fit parameters) with the model were found with predict-first vs without, across the various tokamaks, with metal vs carbon walls, and across rhostar scans. Predict-first experiments did pose a number of challenges in terms of precise controllability of input parameters, and need for a wide range of calculations before the experiment. They also reinforced the need for high resolution profile measurements in the pedestal, and diagnostic improvements have since taken place on a number of devices. Another use of the predictive capability of the model was enabled by noting that, while EPED usually predicts only a single "H-Mode" pedestal solution, there are cases at strong shaping and high density where the 
solution, at a fixed set of input values, can bifurcate into separate "H-Mode" and "Super HMode" (so named because it sits above the H-mode solution in pedestal pressure) solutions. A dedicated set of experiments on DIII-D searched for, and eventually found the Super HMode solution, taking advantage of advances in feedback control of the density profile, which is needed to trace the parameter space path into the Super H-mode region. While these predictive tests of EPED have been surprisingly successful for such a simple model, there are numerous areas where further testing, model improvements, and use of direct turbulence simulations is needed. Particular areas of interest include prediction of the density and impurity profile (pedestal density and $Z_{\text {eff }}$ are taken as inputs in EPED1), rotation profile, and cases where strong turbulence from other mechanisms may be able to constrain both temperature and density gradients, holding the pressure gradient below the KBM critical value, enabling higher pedestals by raising the global $\mathrm{PB}$ constraint.

The second invited talk in the session, by S.Freethy, described using nonlinear gyrokinetic simulations to guide design of new turbulence diagnostics and experiments at ASDEX Upgrade. Existing non-linear gyrokinetic simulations in combination with a synthetic diagnostics allowed real world predictions about the amplitude and frequency spectrum of temperature fluctuations. Moreover, GENE was able to predict whether or not these fluctuations would be measurable with a given experimental setup in a range of L-mode and H-mode plasmas. This motivated the design and construction of a Correlation ECE (CECE) diagnostic to measure them. An existing optical arrangement was used for the CECE and temperature fluctuations were measured on ASDEX Upgrade for the first time. However, in other plasmas where fluctuations were expected, none were seen. The optical arrangement was suspected as the cause and careful modeling of the focusing mirror revealed the beam spot size to be larger than thought. A scan of the beam radius with the GENE synthetic diagnostic showed that the new beam spot size was sufficient to explain the results. With this knowledge, the frequency spectra of the measured fluctuations showed excellent agreement in absolute units to the GENE validation simulations in an ECRH heated Deuterium L-mode discharge. Further predictions have been made for the phase angle between temperature and density fluctuations, which will be measured with a newly commissioned diagnostic at ASDEX Upgrade in the coming campaign.

The third invited talk in the session, by J.Citrin, demonstrated how "training" neural networks on reduced transport models was possible, to extend transport predictions to timescales relevant for fast off-line discharge optimization and realtime model-predictive control. The emulation of quasilinear transport models with machine learning techniques is promising for circumventing the conflicting constraints of accuracy and tractability in turbulent transport modelling. A proof-of-principle neural network transport model was presented, relevant for ITG transport and including 4 input parameters: $\mathrm{R} / \mathrm{L}_{\mathrm{Ti}}, \mathrm{T}_{\mathrm{i}} / \mathrm{T}_{\mathrm{e}}, \mathrm{s}$ and $\mathrm{q}$. The model was trained on a dense multi-dimensional array of QuaLiKiz output, and includes ion heat flux, electron heat flux, particle diffusivity and pinch. The regularized neural network output provides flux outputs within a CPU ms, $\times 10^{6}$ faster than the original QuaLiKiz model (which it emulates), and $\times 10^{12}$ faster than nonlinear turbulence modelling. The proof-ofprinciple model was validated within the CRONOS integrated modelling suite against a JET standard H-mode for both heat and particle transport. Within the RAPTOR code, the neural network transport model provides faster-than-realtime profile predictions for ITER. Extensions to higher input dimensionality (10D) are ongoing, and will include ITG, TEM, and ETG turbulence. Further extensions to the full input dimensionality case ( 20D) are planned, using quasilinear-GENE flux predictions, and basing the training set on a new multimachine profile database being set up for this purpose.

Applying reduced transport models to interpret experimental results and plan new experiments was presented by the three contributing authors summarized below. 
At JET and ASDEX Upgrade, selected tokamak plasmas have been analysed by H.Doerke with the gyrokinetic code GENE in order to extend the knowledge base of the physics effects behind experimentally observed confinement scalings. When built-in into simplified modelling tools, these studies will increase predictive capability. In an ASDEX Upgrade noninductive discharge, ion temperature peaking is under-predicted by TGLF. Including NBI fast ions reduces ITG growth rates and thus likely plays a role for increased ion temperature peaking. Beyond the confirmation of high beta increasing the ion temperature peaking at inner radii, the analysis of a power scan at JET has been extended. Neither increased beta, nor increased flow shear change the ratio of particle to heat flux, so that the increased NBI particle source at higher power is identified as the main player in the observed increase of core density peaking. The study of the impact of collisionality and co-moving frame physics, as well as a direct comparison between a discharge at low and high power are still ongoing.

The predictive capabilities of the reduced MMM7.1 models have been tested by A. Pankin for the high beta poloidal discharges in DIII-D. These discharges represent a new attractive operational scenario in the tokamak because they are characterized by the steady state operation and have reduced requirements for the external current drive as well as NBI torque. The objective of this study is to identify the improvements needed in the components of the MMM7.1 model for the predictive modeling of high beta poloidal discharges. The DRIBM component of MMM7.1 for the drift resistive inertial ballooning modes, the ETG component for the electron temperature gradient modes and the Weiland component for the ion temperature gradient modes, trapped electron modes and some other MHD modes have been investigated. The DRIBM component is found to strongly overpredict the anomalous thermal fluxes in these discharges, which can be partially explained by the fact that the alpha stabilization effect is not yet taken into account in the DRIBM component. The Weiland and ETG components applied together yield reasonable agreement with the experimental profiles with the exception of the electron ITB region. The electron transport in the Weiland component is found to be overpredicted due to a strongly unstable electron mode in ITB. The predicted electron thermal transport is currently being verified using gyro-kinetic codes. The roles of $\mathrm{E} \times \mathrm{B}$ flow shear and Shafranov shift stabilization effects have been also tested for these discharges. It is found that the $\mathrm{E} \times \mathrm{B}$ flow shear plays a relatively weak role in quenching of anomalous transport. In contrast, the Shafranov shift plays a dominant role in the quenching the transport.

To increase confidence in the use of reduced turbulence transport models such as TGLF for routine experimental prediction, a validation effort has been carried out against the LOCSOC database collected at AUG and reported by E. Fable. A long-standing phenomenological puzzle, the LOC-SOC features robustly observed changes in plasma confinement time as the density increases, with associated a number of interesting behavior of the plasma such as nonmonotonic changes in the density peaking, flips in the intrinsic rotation profile, and (mostly speculated) transition from TEM to ITG-type turbulence as the density is increased. Moreover, the critical density for the LOC-SOC transition scales mostly with the plasma current, giving a characteristic non-dimensional ne/Ip parameter (as observed in AUG). Modeling using ASTRA + TGLF reveals that the complexity is very real. Linear turbulence spectra show indeed transition between TEM and ITG turbulence as the density is increased, but the transition(s) happen at different radii at different density, giving this a non-local (in radius and density) behavior that does not translate directly in the LOC-SOC behavior of the confinement time. The latter instead is basically determined by the increased ion transport via destabilization of the ITG and the interplay of this with the increased Te/Ti coupling via classical equipartition. As such, despite the fact that there is a common actuator (the density as a knob to force the LOC-SOC transition), the phenomenology is not directly connected to a single physical mechanism (i.e. the TEM-ITG transition), but links different players: 
equipartition, ITG destabilization, and (not to be forgotten) also the edge which instead does not show any rollover in the confinement time. Nevertheless, the density peaking is directly linked to the TEM/ITG transition as already confirmed in previous studies. The intrinsic rotation profile instead still requires a complete interpretation with respect to fundamental mechanisms.

Some talks from other sessions also covered the theme of the "predict first" effort in the TTF community, and give good motivation to include a "predict first" session again at the next TTFs. For example, the ELMFIRE full-f gyrokinetic code has been used by S.Leerink to make detailed predictions of GAM effects in FT-2 tokamak. The GAM was found to have a large effect on measured Doppler Reflectometry spectrum, and this motivated new studies with Enhanced Scattering reflectometry. This example shows that there are many opportunities to "predict first" whenever groups are already engaging in detailed validation efforts with nonlinear gyrokinetic codes. The base line simulations used in a validation efforts can indeed be further used to motivate new measurements and new experiments.

The discussion at the end of the session was stimulating and spirited. Participants debated the cost/benefit of the approach, and of using advanced and often computationally expensive models to guide design. In many cases where reduced models cannot predict the details of interest (e.g. the fluctuation level of the turbulence), a full model prediction may be needed. The group discussed how one could use codes ahead of an experiment to predict large, unambiguous changes in turbulence/transport, and in some cases, to predict new previously unmeasured quantities. A major part of the discussion revolved around the issue of sensitivity, error analysis and uncertainty. Related to this, the group discussed the challenge of determining the uniqueness of results, namely, how does one discriminate between models? As part of all these issues, the group tended to agree that sharing frameworks/analysis tools developed from validation efforts was important, and that "predict first" was part of the validation cycle.

\section{Electron heat transport and multi-scale integration}

Throughout the last two years, the question on a correct and comprehensive assessment of the electron heat flux - particularly, originating from sub-ion-gyroradius scales - has regained such a momentum that a dedicated session was called for. Already more than a decade ago, it had first been shown by means of (single-scale) simulations limited to electron gyroradius scales that stand-alone electron temperature gradient (ETG) driven turbulence can drive substantial electron heat transport. Later, simplified reduced ion-to-electron mass ratio simulations self-consistently covering both ion- and electron-gyroradius scales could confirm these findings even in a bath of large-scale turbulence. Furthermore, experimental evidence for high-k fluctuations had been established.

However, more comprehensive simulations had not been feasible until recently - amongst others due to the enormous computational effort required. By now, new and challenging insights are available triggering various discussions, e.g., on cross-scale effects, saturation mechanisms and scaling laws. The former were widely covered in the session and will be detailed in the following.

N. Howard presented transport comparisons of electrostatic gyrokinetic multi-scale simulation results performed with the GYRO code with Alcator C-Mod L-mode measurements. Similar to the above simplified setup results, (partial) electron-turbulence suppression in the presence of strongly driven ion-scale turbulence could be found. However, it became clear that reduced mass ratio simulations turn out to be insufficient for proper quantitative assessments and for matching the experimental transport levels in some conditions. Furthermore, a comparison with ion-scale restricted simulations revealed a dramatic change in experimental situations where the ion temperature gradient (ITG) driven 
turbulence is near marginality. Here, the small-scale ETG driven streamer-like structures seem to weaken the zonal flow (ZF) shear generation - the latter being typically considered the main saturation mechanism for ITG turbulence. Consistently, the ion heat transport is substantially increased in a multi-scale simulation. This increase in low-k turbulence is also found to some degree in the electron heat channel. However, here, the additional ETG contribution is more relevant explaining a factor 10 increase compared to the ion-scale simulations. All in all, the flux-gradient relationship may be significantly altered by crossscale coupling in such scenarios. On-going studies seem to confirm these findings in selected C-Mod ELM-y and DIII-D ITER baseline H-modes which suggests that such multi-scale effects should be considered in ITER performance predictions.

Similar small-scale suppressions by strongly driven large-scale turbulence and ion-scale turbulence enhancements by ZF weakening had also been identified by S. Maeyama with the GKV code previously. Here, Cyclone Base Case inspired parameters had been employed together with a realistic mass ratio. Contrary to the previous presentation, electromagnetic field fluctuations had been included as well and been used to stabilize the ion-scale turbulence in one of the cases. The various cross-scale effects or nonlinear mode couplings, respectively, have been analyzed in detail by sophisticated subspace transfer diagnostics. It could be shown that short-wavelength ITG eddies distort ETG streamers while electron-scale turbulence has a damping effects on short-wave-length zonal flows. Finally, preliminary results for the interaction between large-scale microtearing mode (MTM) and ETG turbulence were presented which indicated that MTMs may be partially suppressed by ETG turbulence. This seems to be linked to ETG modes destroying the radially localized currents of MTMs.

A third presentation along these lines has been given by N. Bonanomi addressing the role of small-scale turbulence and cross-scale interactions for the electron heat transport in JET. By analyzing a large JET database at low/high NBI and low/high ICRH power deposition, a correlation of the electron temperature gradient with the ion-to-electron temperature ratio multiplied by the effective charge could be identified which can be related to the linear ETG threshold. A nonlinear study has been started with the GENE code, which recovers the above discussed small-scale turbulence reduction due to large-scale turbulence at low high-k drive in JET. Increasing the ETG drive yields significant small-scale transport contributions and improves the agreement with the experimental findings.

Another aspect and a different kind of multi-scale interaction has been highlighted in G. Colyer's presentation. Here, a collisionality scaling of the electron heat transport found in MAST plasmas where the ion heat transport is found close to the neoclassical value could be reproduced with GS2 simulations focusing on ETG turbulence with adiabatic ions. Crucial for these results were long-time simulations which properly capture the nonzonal and zonal mode interactions and the final state of the latter which represents a fine-scale ZF pattern that prevents the small-scale streamer formation. The heat flux scaling can then be linked to the collisionality dependence of the collisional damping of the zonal modes. Similar patterns as in the MAST cases could also be recovered by N. Howard when reducing the ITG below marginality. With large-scale turbulence being driven, their turbulence structures seem to prevent the establishment of fine-scale ZFs. In any case, simulations should be carefully checked for saturation of the ZF component.

Finally, a validation study for the new TGLF quasilinear saturation rule (SAT1) that aims at capturing the zonal flow mediated cross-scale coupling has been presented by S. Smith. Here, scans in the ECH heat deposition in DIII-D L-modes at two different radial positions have been employed as reference scenarios. It could be found that TGLF can reproduce the trend in the electron heat flux if the relevant scales are considered. However, the new saturation rule including cross-scale coupling effects appeared to not always match the experiment more closely than the old (SAT0) model. Furthermore, no particular coupling 
effect could be observed in the ion heat channel which furthermore seems to be difficult to match although SAT1 had been adjusted to the multi-scale simulations performed by $\mathrm{N}$. Howard. The talk hence concluded with the request for further exploration of trapped electron mode (TEM) regimes which seem to be relevant in the scenario at hand. Additionally, it was emphasized that the saturation rules still require further revision to correctly assess the crossscale interactions.

The need for building a larger international data base of gyrokinetic multi-scale simulations was also highlighted in the following discussion round. While this would certainly be helpful to improve quasilinear models, it could also clarify in which situations computationally much cheaper single-scale approaches would be sufficient. Previous gyrokinetic validation work has mainly focused on ion-gyroradius scales and success in this context points to the existence of parameter regimes where either cross-scale couplings or even high-k contributions are negligible such that multi-scale simulations could be avoided. Besides the so far mainly studied variations of the relative drive of the ion and electron gyroradius scales, it would also be interesting to investigate the multi-scale aspects, e.g., at various radial positions - particularly, near-edge - and for different hydrogen isotopes or impurity concentrations. Another question to be addressed while extending such a data base is whether all relevant cross-scale interactions have already been identified and whether they can be captured with the established analysis tools.

For ETG turbulence, the parameter space where high-k ZF structures evolve on long time scales and become the dominant saturation mechanism rather than the traditionally considered secondaries should carefully be determined as well.

A question to the community is also whether multi-scale simulations could be validated beyond transport comparison. The modification of density fluctuation power laws at wave numbers below but near the ion-gyroradius scale by small-scale turbulence may, for instance, be close to the wave number range accessible to present-day Doppler reflectometry systems.

Finally, the idea whether cross-scale coupling effects could be included in single-scale simulations, e.g., by developing large eddy simulation (LES) or mean-field approaches was briefly discussed.

\section{Understanding power decay length in the SOL}

The power of a tokamak is exhausted to the divertor target plates via the scrape-off layer (SOL) region. Heat and particles exhausted from the core flow rapidly along the magnetic field lines of the open SOL flux surfaces to strike the target plates. These must therefore be designed to carry large thermal loads that, in next step fusion devices, are at the edge of or above what can be handled by today's materials. As a matter of fact, heat load on the vessel is thought to be among the most crucial problem that fusion is facing today, possibly a showstopper for the entire program. A key parameter that determines the power load on the vessel is the width of the SOL region, i.e. its power decay length. This depends to a large extent on the ratio of transport of heat and particles across magnetic field lines to that along them. Neither of these processes is completely understood, so predicting the divertor heat loading for ITER or DEMO has large associated uncertainties.

The session focused on the understanding of the power decay length in the SOL, with the goal to introduce the problem to the non-experts and discuss our current understanding of the mechanisms that regulate the SOL power decay lengths. The session started with three invited talks, followed by two contributed talks and a discussion. Six posters were also presented.

An introduction to the subject was given by B. Lipschultz during the first invited talk of the session. He pointed out that divertor research and modeling gives us reasonable confidence that the current conventional divertor configuration will likely handle the power loads of ITER, but puts into question that the same can be attained in the next step fusion 
devices. In fact, from contemplating DEMO concepts, it is clear that the parallel-to-B heat flux density towards the divertor, could be significantly larger than ITER, raising concerns for attaining and utilizing detachment and the resulting reduction of peak heat flux at the divertor to below engineering limits. B. Lipschultz then pointed out and illustrated the two paths that are being pursued to address this issue: a) Lower the heat flux through core radiation to levels similar to that predicted for ITER; and b) Use 'alternative' divertor geometries to enhance the capability of the divertor plasma itself to further lower the peak heat flux, reaching the target through enhancing cross-field transport and/or more radiative and neutral losses.

The following invited talks by F. Nespoli and B. LaBombard pointed out experimental evidences that clarify the plasma turbulent dynamics in the SOL. These measurements were focused on one of the simplest tokamak magnetic configurations, a plasma with circular magnetic flux surfaces limited on the high-field side wall. Even this simple configuration, a stepping-stone for more complicated geometries, exhibits interesting features that highlight the complex SOL plasma dynamics that underlies the power decay length. In fact, the SOL of inner-wall limited plasmas is known to exhibit two separate regions, namely the near SOL, typically a few millimetres wide and characterised by steep radial gradients, and the far SOL, typically some centimetres wide and with flatter radial profiles. The 'narrow heat flux feature' has recently gained attention because of its importance for ITER start up and ramp down phases. F. Nespoli described new experiments performed in TCV to study the physics of the near SOL. The near SOL is observed for the first time to disappear at the limiter in deuterium plasmas for low currents or high density, i.e. for high resistivity. The strength of the near SOL is shown to be correlated to the presence and strength of non-ambipolar currents flowing to the limiter, and both decrease for increasing resistivity and eventually vanish. The same behavior is observed in helium plasmas. This suggests the suppression of turbulence given by the shear of the $\mathrm{E} \times \mathrm{B}$ velocity as a cause for the formation of a near SOL.

B. LaBombard pointed out that experimental results on Alcator C-Mod, spanning back a number of years, indicate that the narrow feature in inner-wall limited plasmas has actually been masquerading as a prevalent feature seen in the SOL for a very long time. New measurements on Alcator C-Mod, aided by a Mirror Langmuir Probe (MLP) diagnostic, reveal that this narrow feature and its inverse scaling with poloidal field is more ubiquitous to the SOL than previously thought - it is present in H-modes, L-modes, inner-wall limited and diverted plasmas. This means that a physics model that successfully explains the detailed structure of any one of these SOL would likely apply to many. The plasma potential profile is found to peak near the LCFS in both limited and divertor discharges. Outside the LCFS, a positive radial electric field is observed, consistent with the expected ambipolarity constraints imposed by sheath physics and parallel electron force balance, making the plasma potential proportional to the electron temperature. Apparently, in response to this electric field, the plasma rotates toroidally: poloidal projections of parallel flow and $\mathrm{E} \times \mathrm{B}$ flow are found to cancel. Inside the LCFS the radial electric field becomes negative, with a magnitude and direction that approximately cancels the ion diamagnetic velocity. The result is a robust $\mathrm{E} \times \mathrm{B}$ shear layer, roughly centered at the LCFS. Shearing rates comparable to the ideal ballooning growth rate are measured. Fluctuations appear to be 'drift-like' in the near SOL propagating at the sum of $\mathrm{E} \times \mathrm{B}$ and electron diamagnetic velocities - and 'interchange-like' in the far SOL - with 'blobs' propagating at the time-averaged $\mathrm{E} \times \mathrm{B}$ velocity. The 'breakpoint' in the slope of the SOL profiles that defines the 'near' and 'far' SOL is well correlated with the location of changeover of this propagation velocity. Measurements on the low-field side SOL have been compared with those on the high-field side, making use of CMod's inner-wall scanning probe, particularly in double-null discharges. These measurements illustrate dramatic differences in the SOL transport, including a 'quiescent' SOL on the high field side with essentially zero turbulence and extremely sharp electron pressure profiles in 
double null configurations. In this case, the high field side SOL appears to be entirely composed of a near SOL gradient. The electron pressure e-folding length there is very similar to that seen on the low-field side SOL. Both appear to scale inverse with poloidal magnetic field.

Two contributed orals, focused on our recent progress in the understanding of the SOL physics, concluded the oral session. J.J. Rasmussen described the use of a two-dimensional fluid model that includes finite ion temperature and is implemented in the HESEL code to study the dynamics of filaments and the associated transport in the SOL region. A clear difference between ion and electron temperature in the filaments and in the background plasma is observed, the ion temperature being typically from two to four times larger than the electron one. The majority of the electron energy is drained to the divertor region by the highly efficient electron heat conduction along magnetic field lines. The ion energy, for which the parallel heat conduction is much weaker, has a much broader SOL profile following the density profile. At higher density, with "shoulder" like SOL-profiles, the energy deposition at the first wall will be enhanced and the ion contribution to the power deposion at the divertor target will be significantly broader than the electron contribution.

C. Baudoin reported on the use of a reduced 2D version of the fluid code TOKAM3X to study the role played by the curvature drift in the transport of particles in the limit of weak collisional and anomalous transport. Both limiter and divertor geometries were studied and compared. In the limiter configuration, the SOL density decay length scales as the square root of the diffusion for high diffusion coefficient, but saturates at a non-zero value for low diffusion coefficient. This value is significantly lower than the one predicted by the heuristic model. Moreover, at low diffusion coefficient, a complex neoclassical equilibrium appears, presenting strong poloidal asymmetry. In divertor configuration, the results are qualitatively similar but of a different order of magnitude due to modification of the flux expansion. In the tested configurations (JET-like shape), the SOL width is found twice as small as in comparable limiter cases.

A discussion followed the oral presentations. The importance was pointed out of addressing the simplest magnetic configurations as a stepping stone towards more complicated scenarios that are considered for reactor relevant conditions. The conditions that allows the use of simplified models, such as fluid, with respect to fully kinetic models were also debated. During the poster session, F. Riva and J. Morales presented the status and recent advancements of the GBS code that is used to model plasma turbulence in the SOL. R. Jorge discussed the development of a kinetic model that includes the collisions at high order and is appropriate to study plasma turbulence in the SOL. M. Held presented numerical simulations of seeded blobs in the SOL based on a four field full-F gyrofluid model, which entails the consistent description of high fluctuation amplitudes and dynamic finite Larmor radius effects, showing that a temperature perturbation increases the maximal blob velocity and that a finite Larmor radius contributes to highly compact blob structures with finite poloidal motion. Finally, two posters focused on blob measurements were presented. S. Garland described the influence of geodesic curvature and magnetic shear on poloidal blob motion in the stellarator TJ-K by using Langmuir probe measurements, while M. Lampert reported on the use of hydrogen beam emission spectroscopy to study the 2D blob dynamics on the KSTAR tokamak.

\section{Role of the SOL in the L-H transition}

It has been observed experimentally that conditions in the SOL and in the divertor can have strong consequences on the confined plasma and more particularly on the access to $\mathrm{H}$ mode. In present day machines, experimental data exhibit a factor $\sim 4$ scatter in the L- to Hmode power threshold, $\mathrm{P}_{\mathrm{LH}}$, compared to predictions of the empirical scaling law. However, 
this scaling does not account for a large number of parameters describing particularities of experimental conditions, especially in the SOL and divertor region. The focus of this session was to define key parameters and physical mechanisms in the tokamak SOL, which strongly affect the L-H transition, from both theoretical and experimental points of view. The session consisted in two invited talks reviewing these key parameters and two orals presenting numerical works on the L-H transition.

A. Chankin presented an overview of the possible mechanisms occurring in the SOL and divertor that have an impact on the H-mode power threshold. He reported on experimental results as well as on numerical simulations that support experimental observations. Wall conditions, magnetic equilibrium, geometry of the divertor (e.g. open or closed for neutrals) or methods of density control contribute all to the L-H transition at a given input power. These parameters are not covered by the $\mathrm{P}_{\mathrm{LH}}$ scaling, but can explain the observed experimental scatter in $\mathrm{P}_{\mathrm{LH}}$. A. Chankin reminded that $\mathrm{P}_{\mathrm{LH}}$ is lower in normal $\mathrm{B}_{\mathrm{t}}$ orientation thanks to a heat flux through separatrix from SOL to core due to $T_{i}$ variation along the separatrix. He also noted that the $\nabla \mathrm{B}$-drift direction has only a small effect on the pedestal but a large effect in the divertor. Then, he pointed out the importance of the divertor geometry effects such as the X-point height and the strike-point position. In JET, MAST, DIII-D, the $\mathrm{P}_{\mathrm{LH}}$ decreases with decreasing X-point height. However, in ASDEX-Upgrade (AUG), experimental observations are not in line with other machines. In C-Mod and JET, the outer strike-point positioned in the horizontal target favors a lower $\mathrm{P}_{\mathrm{LH}}$ with respect to when positioned on the vertical target, pointing out the role of neutrals. The role of $\mathrm{T}_{\mathrm{e}}$ at the outer strike-point, and thus the radial electric field $\mathrm{E}_{\mathrm{r}}$ at the separatrix, was also detailed. Experimental observation of peaked $\mathrm{T}_{\mathrm{e}}$ target profiles and lower $\mathrm{P}_{\mathrm{LH}}$ are explained by $2 \mathrm{D}$ fluid simulations through a modeled larger positive $\mathrm{Er}$ at the separatrix. Correlations between $\mathrm{T}_{\mathrm{e}}$ peaking on the divertor, modeled near SOL $\mathrm{E}_{\mathrm{r}}$ and observed $\mathrm{P}_{\mathrm{LH}}$ are clearly established for different working gases (H, D, T), wall materials (C vs. W), divertor geometry (vertical vs horizontal targets), ion grad-B drift direction, etc. Finally, one of the main conclusions of this review talk was that very varied experimental observations leading to modified L-H power threshold can be explain by an identical mechanism, namely the target $\mathrm{T}_{\mathrm{e}}$ profile modification leading to modified $\mathrm{E}_{\mathrm{r}}$ at the separatrix, hence modified $\mathrm{E} \times \mathrm{B}$. This proposed explanation should lead to more systematic measurements of $T_{e}$ on the divertor as well as of $E_{r}$ around the separatrix for further validation.

H. Meyer presented an overview of experimental results in present day devices that have an impact on the access to H-mode. His review focused mainly on the SOL and divertor conditions. He reminded that the progress made in diagnostics enables more accurate and fast observations, that have led to highlight the role of several mechanisms occurring in the confined plasma (dynamics of the shear flows, ion heat flux), but the same consistent picture in the SOL is still blurry. H. Meyer reported recent JET results that shed light on the importance of divertor conditions with a $\mathrm{P}_{\mathrm{LH}}$ decreased by nearly a factor of 2 in configurations limited by the septum at the X-point. Later studies on JET with the septum removed showed that the same reduction is achieved by simply shifting the magnetic configuration downwards, more precisely with the outer strike-point going from a vertical target to an horizontal target configuration. This sensitive dependence on the magnetic configuration in the divertor has since been observed on many devices such as Alcator CMOD, COMPASS, DIII-D, EAST, MAST, NSTX and TCV but not in AUG and so far no consistent picture has emerged. These studies also show an influence of the lower and upper triangularity on H-mode access. Recent studies on JET comparing configurations with both strike points on the vertical targets to configurations with one strike point on the horizontal target suggest that changes to the sheath potential profile could play an important role in altering the near-SOL radial electric field. These changes occur due to the changes in the 
recycling. Indeed, a 30\% reduction of $\mathrm{P}_{\mathrm{LH}}$ has been reported in a metallic environment with $\mathrm{W}$ walls when compared to former C walls in both JET and AUG. Similarly, a reduction by a factor of 2 on $\mathrm{P}_{\mathrm{LH}}$ is observed in NSTX after Li evaporation. The changes of the SOL parallel flow direction on Alcator C-MOD correlate with the differences of $\mathrm{P}_{\mathrm{LH}}$ observed with opposite ion $\nabla \mathrm{B}$-drift direction, indicating that a similar SOL flow is needed to access $\mathrm{H}$ mode. On AUG, MAST and NSTX a change of $\mathrm{P}_{\mathrm{LH}}$ in exact double null configuration is observed, where the most remarkable changes on MAST are happening in the high field side SOL and the inner strike points. However, in all these experiments, the role of the mean field $\mathrm{E}_{\mathrm{r}}$ seems to appear as a common feature in the access to H-mode. An ITPA (International Tokamak Physics Activity) task has been created in the Pedestal \& Edge Physics topical group to focus on the understanding in the $\mathrm{L}-\mathrm{H}$ transition from the SOL and divertor point of view.

In his oral presentation, N. Nace showed the importance of the ion heat flux channel on the edge radial electric field and on the transport barrier dynamics at the transition between open and closed field lines using TOKAM-2D, a reduced bi-dimensional fluid code based on the interchange instability, that can solve consistently the electron and ion energy balances. The focus is made on the contribution of the ion diamagnetic velocity in the polarization drift, i.e., in the ion perpendicular inertia, by adding this term to a generalized vorticity. A link is thus established between the radial electric field and the ion pressure gradient, which seems to be a key feature of the pedestal formation. Varying the ion injected power reveals the existence of a threshold which controls the radial electric field profile and the dynamic of the transport barrier, the ion pressure stabilizing the barrier and its efficiency being increased with the ion heat flux. However, these simulations appear to strongly underestimate this injected power threshold compared to experimental observations, suggesting that additional mechanisms are probably at play to limit the build-up of the barrier. Limitations of the $2 \mathrm{D}$ model are its sensitivity to viscosity, the inner radial boundary conditions, geometrical effects and parallel dynamics. Indeed, a preliminary similar scan realized using the $3 \mathrm{D}$ edge turbulence simulations by TOKAM-3X leads to very different results. At equivalent input power, the formation of much weaker, although non zero, transport barriers is observed.

X. Q. Wu presented a new model of the L-H transition based on the flow shear induced edge instability phase transition. Near the separatrix, the parallel electron motion is strongly impeded and MHD-type modes may appear, such as pressure driven modes, here represented by the interchange mode. In the closed field line region well inside the separatrix, the resistive drift-ballooning mode may dominate. The radial transport driven by this mode is not as strong as the MHD-type modes. So, the L-H transition should be associated with the stabilization of the MHD-type modes near the separatrix. In the model presented, the dispersion relation for the pressure driven instability is derived from a reduced two-fluid model, and the flow shear at the edge is dominated by the diamagnetic drift. Importantly, the two-point model of the SOL transport is applied to estimate the dynamics of edge temperature and density, which is crucial to the power threshold scaling. As preliminary result, the model is able to reproduce a reduction of the turbulence amplitude during a L-H and a H-L transition controlled by the input power (with an increase of the pedestal pressure). The dependence of the threshold power on the pedestal density for the $\mathrm{L}-\mathrm{H}$ transition is obtained with the SOL boundary conditions.

The session was closed by an open discussion. One of the main focus was on the available multiple data from multi-machine experiments that are not sometimes coherent from machine to machine. The priority should be to untangle the main driving mechanisms because it seems that more than one mechanism could be important for accessing the H-mode. Moreover, an effort should be done to compare measurements with existing models in order to confirm or infirm some of them. For this purpose, there is a need for simultaneous SOL and confined 
edge plasma measurements of quantities such as $E_{r}, T_{e}, n_{e}$, flows, etc. Another interesting remark was that the turbulence close to the last closed flux surface is fundamentally different than in the edge region. The question of which turbulence should be suppressed was raised. Finally, modeling should be emphasized and the comparison with experiments is a crucial point for future understanding. The work done under the umbrella of the ITPA to address the $\mathrm{L}-\mathrm{H}$ transition mechanisms from the SOL and divertor regions is going in that direction and hopefully progress will be reported at the next TTF meeting.

\section{Validation of fundamental turbulence properties against turbulence measurements}

A session including four invited and two contributed talks was held, which focused on direct comparisons between measured turbulence characteristics (such as fluctuation amplitudes, frequency spectra, correlation lengths, and correlations between fluctuating fields) and theoretical predictions, particularly through non-linear simulations and including synthetic diagnostics for well-constrained evaluations of the level of agreement. Turbulent transport is often in stiff regimes in tokamaks, where small changes in gradients can correspond to large differences in turbulent fluxes. This results in transport models often being poorly constrained when considering only the relationship between the equilibrium gradient values and the driven fluxes, since there can be a large range of fluxes consistent with experimental uncertainties for measured gradients. Direct comparison between measured turbulence properties and simulations provides a much stronger challenge to transport models and is necessary to understand the predictive value of such models, in particular when extrapolating to future devices.

In the first invited talk, A. Bañón Navarro presented comparisons of turbulence measurements from ASDEX-Upgrade to linear and non-linear GENE simulations. A target H-mode AUG pulse was studied, where ECRH power steps were used to increase the electron temperature and modify turbulence drive terms; linear GENE parameter scans showed that trapped electron mode activity was more strongly driven as a result. Both local and global non-linear simulations were performed. Although small finite $\rho^{*}$ effects (i.e. global size effects) were revealed by differences between the local and global simulations, both were consistent with experimental power balance analysis after modifying the ion temperature gradient used in the simulations by an amount also within experimental uncertainties. Comparisons to electron density fluctuation measurements made with Doppler reflectometry showed that while GENE reproduced the radial increase of turbulence amplitude from the core towards the edge, the amplitudes showed opposite trends with heating power - GENE predicted decreases while the measurements increased. Multi-scale simulations might be necessary to resolve the discrepancy. Predictions were also presented for quantities which were not measured in the experiment, but are expected to be available at AUG in the near future: non-universal power laws were predicted for density fluctuations, depending on radius; electron temperature fluctuation levels were predicted to be above anticipated noise levels for a CECE system; and the cross-phase between density and temperature fluctuations was identified as being insensitive to the ion temperature gradient and therefore a good quantity to use in gyrokinetic validation studies.

The second invited talk of the session, from D.R. Ernst, presented comparisons between DBS (Doppler reflectometry) measurements in DIII-D QH-mode plasmas and non-linear GYRO and GENE simulations, including full-wave modeling of DBS as part of a synthetic diagnostic. ECRH was used to modify kinetic profiles such that density gradient driven trapped electrons modes became the sole instability, and were observed as a set of discrete toroidal modes in measured spectra and reproduced in simulations. Both with and without ECRH heating, GYRO simulations were consistent with transport analysis and experimental uncertainties for particle flux, toroidal momentum flux, electron heat flux, and ion heat flux. 
After including beam curvature effects determined from full-wave simulations, synthetic DBS spectra from GYRO closely reproduced spectral changes observed in the experiment after ECRH was added, including features at the level of discrete mode structure in the spectra due to coherent modes. Predictions of $\alpha$-heated plasmas with similar turbulence drive as the experiment found that $\alpha$-heating would flatten the density profile, reducing fusion power, but that the effect could be counteracted by broadening the q-profile near the magnetic axis to optimize the magnetic shear.

G.R. McKee presented the third invited talk, which compared multi-scale turbulence measurements from BES, DBS, and PCI to non-linear GYRO and quasi-linear TGLF results focusing on target high $\beta_{n}(\sim 2.5-3)$ DIII-D hybrid H-mode plasmas where the rotation was varied by modifying the mix of co- and counter-NBI sources. The experiment found that increased rotation shear elongated turbulent eddies in the direction of the shear flow, increasing the poloidal correlation length, but did not reduce the radial correlation length. Comparisons between density fluctuation spectra constructed from quasi-linear TGLF results and BES measurement show qualitative agreement with experimental trends when rotation shear and the electron to ion temperature ratio were varied. Comparison of synthetic BES predictions from non-linear GYRO simulations showed good quantitative agreement with the experimental spectrum, in independent absolute units. Although good agreement for fluctuation level was found, some modest discrepancies were also identified in comparisons of the spatial correlation function, and a significantly different correlation time.

The final invited talk was presented by L. Porte, showing comparisons between turbulence measurements in TCV with TPCI, CECE, and DBS and simulations with GENE and ORB5, focusing on characteristics of the GAM measured with TPCI. TCV measurements showed the GAM can appear either as a global mode with a constant frequency over a radial range, or in a dispersive condition where the mode frequency varies locally with radius. Local nonlinear simulations were found to reproduce the GAM frequency and propagation direction, while global runs were needed to reproduce the location of the mode. GENE was found to be able to reproduce the transition from global to dispersive GAM activity, and further work was planned to investigate the impact of $\rho^{*}$ on the transition. Further open questions on GAM physics were identified including investigation of the relationship between the GAM and bursts of energy and particle transport at the GAM frequency in global simulations, understanding what sets the frequency of a global GAM, and understanding systematic differences between local and non-local simulations for the frequency of the GAM.

There were two contributed talks in the session. E. de la Cal showed results investigating the response of neutrals to plasma turbulence through 2D measurements of electron density blobs. Comparison of measurements and a 1D neutral model showed better agreement with dynamic neutrals rather than static neutrals, implying that turbulent dynamics of neutrals could be important in the plasma edge. T.A. Carter showed experimental results from high beta LAPD plasmas (up to 15\%) with increasing magnetically driven transport at high beta due to fluctuations in the parallel component of the magnetic field. Observations showed consistency with a new Gradient Driven Coupling instability identified in non-linear GENE simulations.

In the poster session, I. Cziegler presented results on the study of turbulence and flow dynamics in I-mode and I-H transitions in Alcator C-mod, addressing the role of GAM-ZF competition or interaction for I-H transitions in comparison the edge ZF dynamics of the L-H transition. D. Dickinson presented ongoing work on the implementation of electromagnetic gyro-fluid simulations in BOUT ++ , for the goal of non-linear pedestal simulations at much reduced computational cost in comparison to gyrokinetic simulations. J. Hillesheim reported results from JET on the measurement of fine-scale structure in the edge radial electric field consistent with zonal flows, suggesting that the ratio of the turbulence correlation length to 
the width of the edge $E_{r}$ could be important for determining the dynamical regime of edge ZF. J.-w. Kim showed detailed investigation of the properties of statistical analysis techniques used for correlation measurements, finding that the uncertainty for measurement parameters could be determined analytically and verifying analysis of KSTAR BES data. J. Anderson presented work from S. Moradi studying a predator-prey model of coupling between populations of stochastic oscillators, showing how self-organisation and synchronization is achieved in the model, highlighting analogous behavior between drift waves and zonal flows in tokamaks.

Together the invited talks showed that in comparisons across a range of plasmas conditions and measurement techniques it is possible to simultaneously match experimental turbulent fluxes and trends for fluctuation levels, in some cases the absolute fluctuation level and features of fluctuation spectra, and characteristics of saturation mechanisms like the GAM. Several cases of disagreement also pointed to needs to assess the role of multi-scale interaction, implementation of synthetic diagnostics, and an expansion of high-k measurement capabilities. Discussion at the end of the session included consideration of whether the community is focusing on comparing the correct quantities, with it being noted that, while a number of important measurements are made consistently, like fluctuation levels and frequency spectra, there is a lot that could be gained through more use of deeper analysis for

signatures of non-linear interactions like bispectral analysis and wavenumber spectra. The need was also recognized for turbulence and transport model validation studies to progress from the core to the more challenging edge, to better understand pedestal transport and interaction between the plasma edge and the SOL.

\section{Posters on core transport}

D.Hogeweij presented successful simulations of the evolution of $\mathrm{T}_{\mathrm{e}}, \mathrm{q}$ and NTM (mainly 2/1) in TCV using the code RAPTOR under various conditions of NTM triggering \& suppression.

A.Lebschy discussed the characterisation of the core poloidal flow structure at ASDEX Upgrade. It was found that for the observed dataset $v_{p o l}$ agrees within the error bars with neoclassical predictions. $\quad \mathrm{v}_{\mathrm{pol}}$ measurements across the LOC-SOC transition show slightly smaller values than expected from neoclassical theory in LOC and at the edge.

H.Kaang presented a poster on electromagnetic fluctuation effects on residual stress and momentum transport in ITG turbulence. EM effects on intrinsic rotation generation are expected to be important.

P.Niskala presented gyrokinetic investigations of isotope effect on particle transport at FT-2 using the particle-in-cell code ELMFIRE. The particle confinement improvement seen from $\mathrm{H}$ to $\mathrm{D}$ plasmas is reproduced by simulations. An important role is ascribed to the crossphase between turbulent density and velocity fluctuations.

S.-P.Pehkonen described the experimental determination of intrinsic torque in ASDEX Upgrade, as a part of a multi-machine scaling. The NBI modulation was modeled both with a simplified 1.5D transport equation and a 1D Onion skin model. The data analysis method was found to play a large role in the shape of the obtained intrinsic torque profile and edge value. Determining the best method requires future benchmarking experiments. In addition, unknown contributors, difficult to quantify, such as modulation in the confinement time, may skew the results heavily and require further analysis.

S.S. Kim presented tokamak ITG turbulence simulations using the BOUT++ framework in the core region. The Landau damping operator has been implemented and linear benchmark exercises done. Rosenbluth-Hinton residual zonal flows \& neoclassical poloidal flows have been included. Nonlinear simulations are underway focusing on the role of ZFs in turbulence suppression. 
P.Mantica presented recent results from JET where ion destiffening could be obtained with fast ions from just high ICRH power, with minimum NBI and rotation. She also presented an exercise of validation of TGLF (sat0 and sat1) and QLK against JET ion stiffness measurements at $\rho_{\text {tor }}=0.33$. TGLF and QLK for different reasons match the experimental points without the need of non-linear EM stabilization, at variance with GENE and GYRO: QLK due to overestimate of the $\mathrm{E} \times \mathrm{B}$ stabilization at inner radii, TGLF because of too low ion stiffness, especially when impurities are taken into account.

F.N. de Oliveira described modeling of ICRF power deposition, ICRF driven fast ions and transport levels in AUG discharges with large core ion temperature gradients with combined NBI and ${ }^{3} \mathrm{He}$ minority ICRF heating. GENE linear simulations show that ICRF-driven fast ions can reduce the linear ITG growth rate and shift the KBM threshold in these plasma conditions, thus suggesting their key role in explaining the experimental results. Non-linear simulations are in progress.

I.Pusztai discussed turbulent transport of $\mathrm{MeV}$ range cyclotron heated minorities, created by three-ion minority ICRH scheme, as a mock-up of fusion alphas. Using gyrokinetic tools handling non-Maxwellian species and coupled radial-energy transport simulations to study the effect of turbulent transport on energetic ions, it is found that heated ions are more sensitive to turbulence effects than injected ions and alphas.

A.Salmi showed that density peaking increases with reduced collisionality in JET 3-point dimensionless collisionality scans in Deuterium and in Hydrogen. Modelling of the mid-point D discharge, including modeling of gas puff modulation, indicates NBI fuelling as the largest factor in the peaking. New experiments in Hydrogen utilized $18.5 \mathrm{~Hz}$ strike point sweeping to probe the recycled neutral ionization source inside the separatrix. Data analysis is ongoing.

Y.Zhang presented a transport study on the 3 sub-states marking the evolution of the Quasi-Single Helical state on RFX-mod. He discussed changes in position and strength of the eITB region and their consequences on confinement.

J.Rasmussen showed that considering a model including the effects of turbulence spreading and the impact of the turbulence intensity profile onto the residual stress, it is possible to demonstrate fast cold pulse propagation and cold pulse polarity reversal.

\section{Posters on pedestal transport}

A large activity in the transport community is now focused on modeling and understanding the transport limiting the pedestal build-up.

For example, in JET-ILW, pedestals away from the peeling-ballooning limit are reported. M. Kotschenreuther, D. Hatch and coauthors are proposing an explanation using the gyrokinetic code GENE, where the dilution impact in a $\mathrm{C}$ wall environment is explaining more stable pedestal, see the section above on D. Hatch's oral.

In EAST (R. Chen's poster), NBI H mode, ELMs are required to avoid high Z core concentration. ELMs triggering and control is reported. A Quasi-Coherent mode observed in the small-ELM-dominant plasma regime is accompanied by electromagnetic oscillation and has some similarities with Alfven eigenmodes.

On DIII-D (Xi Chen's poster), an H mode without ELMs is triggered when the NBI torque is reduced in the balanced double null shaped plasma. ELMs reappear if the NBI power is further increased. In the ELM free phase, the turbulence limiting the pedestal build up is analyzed.

$\mathrm{H}$. Jhang is exploring the non-linear dynamics of the ELM onset beyond the standard linear peeling-ballooning model. The nonlinear findings are that zonal flows are playing an important role in pedestal collapse dynamics. 
KSTAR H modes with NBI are analyzed in S. H. Ko's poster. The focus is on the ion temperature and rotation correlation which is found to be larger for smaller NBI torque suggesting an important role plaid by an intrinsic rotation in the pedestal.

Finally, a new tokamak environment is coming online, WEST, and some specificities are put forward in C. Bourdelle's poster as opportunities for the transport community: the full W environment, the larger aspect ratio (5 to 6), a dominant electron RF heating, and low torque plasmas.

\section{Conclusions}

This section provides a brief summary of all of the sessions of the workshop, and outlines the main open issues and the priorities for future work.

In the session "Progress towards full-F kinetic turbulence simulation", the current status of full-F gyrokinetic simulations was discussed. Thanks to dramatic increase of computational resources, the capability and utility of full-f gyrokinetic simulations have been greatly expanded. The highlights of such new capabilities are kinetic electron models and experimentally relevant boundary conditions in edge plasmas, while electromagnetic turbulence simulations are still prohibitive. In addition to physic studies on nonlocal transport and meso-scale structure formations, which are unique features in global full-F models, comprehensive validation studies were presented, and the accuracy of current full-F models was discussed. Applications of full-F gyrokinetic simulations to ITER relevant issues such as the divertor heat load width, isotope effects on the confinement, and rotation changes induced by electron heating modulation were also presented. Future priority issues in this session could be the development of electromagnetic full-F models and more systematic validation studies against larger devices, which will be supported by advances in computing technologies.

The session dedicated to impurity transport emphasized that for medium $\mathrm{Z}$ impurities (e.g. $\mathrm{B}, \mathrm{C}, \mathrm{N}$ ) the hollow profiles observed experimentally in various conditions in AUG and JET are not accurately predicted by the simulations. The sum of neoclassical transport from driftkinetic simulations and turbulent transport from gyrokinetic simulations tends to systematically overestimate the impurity peaking in the conditions where hollow profiles are observed. Given the interest of hollow impurity profiles for a reactor operation, understanding this discrepancy is a high priority issue. Mechanisms involving the interplay between neoclassical processes and turbulence are currently under investigation. The impact of sawteeth and other MHD modes on impurity transport remains relatively unexplored (in comparison to neoclassical and turbulent transport) and would also deserve further attention, both theoretically and experimentally.

Concerning the impact of impurities on energy confinement, the overall picture appears to be quite complex with impurities affecting the power balance directly and indirectly by changes in the radiation losses, the impact of dilution on the total pressure, changes of the Ohmic power (via resistivity) or NBI power deposition (via the electron density profile), ELM frequency, pedestal stability and turbulent transport (collisionality, safety factor profile, main ion dilution, $\left.T_{e} / T_{i}, \ldots\right)$. The number of mechanisms and couplings involved makes this problem rather challenging and a close interaction between the experiments and the modelling seems necessary. The impact of impurities on transport in the pedestal is one of the key issues. On this front, the reduction of the ITG turbulence by main ion dilution was proposed to explain the behaviour of the JET pedestal with the ITER-like wall.

As discussed in the 3D session of the workshop, significant experimental and numerical progress has been made on understanding impurity transport dynamics in stellarator equilibria. It has also been shown that the phase and amplitude of the applied RMP field in the DIII-D tokamak can result in significant differences in the core confinement. These 
confinement changes may results from differences in the coupling between intrinsic 3D fielderrors and the applied RMP field as well as subtleties involving the coupling of ideal kink modes and resonant magnetic islands produced by the plasma response to the external RMP fields. A high priority for future studies is to better understand interactions between the ideal and resistive MHD plasma response especially when multiple toroidal and poloidal modes are excited by small intrinsic and applied 3D magnetic field perturbations in high performance tokamaks. Density pump out during the application of RMP fields remains an important physics topic. New experiments on TEXTOR and RFX-mod have verified earlier observations of convective cell formation associated with magnetic islands that dominate the local heat and particle transport. Future work in this area could lead to a predictive theory of density pump out in ITER relevant plasmas that would be beneficial for optimizing the pedestal density during ELM suppression. Significant progress has also been made on understanding the structure of the radial electric field and its impact on the pedestal density pump out during the application of RMP fields in tokamaks. In addition, a 3D plasma fluid and Monte Carlo neutral transport code, EMC3-EIRENE originally developed for stellarators, with the inclusion of the resistive MHD plasma response from the M3D-C1 code has been used to study transport in DIII-D H-modes during the application of the RMP. Work in this area of research is important for understanding the impact of the RMP field on divertor heat and particle recycling flux. Continued validation of the physics models in these combined codes is essential for developing a predictive model for the plasma response to the RMP field in ITER.

This year the EU-TTF group held a new session on "Predictive experimental design", or "predict first", for short. While this session discussed the use of integrated models to develop simulations of the time evolution of a tokamak discharge, the session's invited talks highlighted cutting-edge work being done via less integrated, albeit no-less rigorous, techniques. The goal of the session was to provide examples of the use of stand-alone theory based codes (e.g. such as EPED or gyrokinetic codes like GENE and GYRO) to aid in the experiment preparation phase. The codes are used by experimental leaders to guide selection of plasma parameters, types of shots, to motivate new diagnostic development - all with the goal of predicting new turbulence and transport behaviour before it is measured. Based on a very active discussion during the session, it was clear that many "predict first" efforts are of great interest in the fusion community and the topic should remain in the focus of the TTF in future meetings. The discussion also indicated that the community sees "predict first" as part of on-going validation efforts, and as such, the group emphasized the critical importance of error and sensitivity analysis, both for predictions and postdictions.

A particularly valuable session (not only) in terms of computational time indirectly involved was dedicated to "Electron heat transport and multi-scale integration". Very recent and thrilling results regarding cross-scale interactions between different ion- and electronscale turbulence types, new insights on the possible saturation of ETG via fine-scale zonal flows, and first comparisons of a new quasilinear tool including cross-scale effects with experimental findings were presented. It became clear that further simulations should urgently identify the parameter regimes where meaningful results can only be obtained with full multiscale simulations as opposed to those where (much cheaper) separate scale approaches are sufficiently realistic - the latter possibly involving new models approximating cross-scale effects to lowest order. A possible key parameter could, for instance, be given by the proximity to marginality. While performing such simulations, special attention should be paid to the long-time behavior as the aforementioned fine-scale zonal flows may only be established after several hundred (small-scale) turbulence correlation times. Such studies may also help to further improve lower-hierarchy models, which are found to perform much better but still have problems to assess all aspects of small-scale contributions. 
Since heat exhaust constitutes one of the most crucial issues that fusion is facing today, a session of the meeting was dedicated to the power decay length in the SOL. The session dealt with the understanding of the mechanisms that regulate plasma transport in the periphery of fusion devices. Because of the large fluctuations and related variation of plasma properties, in particular collisionality, and because of the multi-physics nature of the tokamak edge, where the plasma interacts with neutral atoms, impurities, and the solid walls of the vessel, the development of a proper plasma model for the tokamak periphery is not accomplished yet. The development of such a model should become a priority for the field. The meeting pointed out that this model should be first applied to study simple tokamak magnetic configurations, such as the limited one. In fact, the physics observed in limited plasmas is shown to display similar features as the ones in diverted configurations. For instance, the narrow heat feature in proximity of the last-closed flux surface and the associated shear layer, the profile of the electrostatic potential, the nature of the instabilities driving turbulence in proximity of the last-closed flux surface and at some distance from it, the pattern of the plasma flows, show striking similarity between limited and diverted discharges. These are the phenomena on which the attention should be focused, and analysing a simple configuration will make it easier to advance the understanding of these main physics processes also when they are at play in reactor relevant scenarios, allowing key progress towards reliable predictions for the ITER and DEMO exhaust.

During the session dedicated to assessing the role of the SOL in the L-H transition, the main focus was to define the key parameters and physical mechanisms that strongly affect the access to H-mode. Experimentally, there is clear evidence that local parameters (wall conditions, divertor geometry, X-point height, etc.) contribute to the L-H transition at a given input power. Unfortunately, no clear picture has emerged yet with some discrepancy and even contradictions in experimental observations from machine to machine. Nevertheless, it seems that the role of the radial electric field via the peaking of electron temperature at the divertor target is a predominant mechanism in accessing the H-mode. Recent 2D fluid simulations have reinforced experimental observations showing that the L-H power threshold is strongly correlated to the target $\mathrm{T}_{\mathrm{e}}$ profile modification leading to a modified $\mathrm{E}_{\mathrm{r}}$ at the separatrix. Therefore, there is a priority to perform such measurements as well as simultaneous SOL and confined edge plasma measurements of quantities such as $E_{r}, T_{e}, n_{e}$, flows, etc., and a newly created, dedicated ITPA task is going in that direction. The transport barrier dynamics can be now simulated by 2D (and even 3D) fluid codes at the transition between open and closed field lines. Preliminary results show the importance of the ion heat flux channel on the edge radial electric field. A new model based on the flow shear induced edge instability phase transition and applying the two-point model of the SOL transport to estimate the dynamics of edge temperature and density is able to reproduce a reduction of the turbulence amplitude during a L-H transition controlled by the input power. The continuation of such works is strongly encouraged, modeling should be emphasized and the comparison with experiments is a crucial point for future understanding.

The session on "Validation of fundamental turbulence properties against turbulence measurements" showed that in comparisons across a range of plasmas conditions and measurement techniques it is possible to simultaneously match experimental turbulent fluxes and trends for fluctuation levels, in some cases the absolute fluctuation level and features of fluctuation spectra, and characteristics of saturation mechanisms like the geodesic acoustic mode. Several cases of disagreement also pointed to needs to assess the role of multi-scale interaction, implementation of synthetic diagnostics, and an expansion of high-k measurement capabilities. Discussion at the end of the session included consideration of whether the community is focusing on comparing the correct quantities, with it being noted that while a number of important measurements are made consistently, like fluctuation levels and 
frequency spectra, there is a lot that could be gained through more use of deeper analysis for signatures of non-linear interactions like bi-spectral analysis and wavenumber spectra. The need was also recognized for turbulence and transport model validation studies to progress from the core to the more challenging edge, to better understand pedestal transport and interaction between the plasma edge and the scrape off layer.

Various experimental tools and techniques are being developed to study toroidal and poloidal rotation and compare with theory predictions, particularly to study intrinsic rotation, whose large experimental values cannot yet be matched by local simulations. Validation of gyro-kinetic and quasi-linear models for heat and particle transport against experiment is a continuous international effort. Some discrepancies in the quantitative effect of stabilizing mechanisms such as $\mathrm{E} \times \mathrm{B}$, impurities, fast ions have been found between quasi-linear and gyro-kinetic models. A stabilizing effect of fast ions on ion heat transport has been observed also in AUG, in addition to JET. Work is on-going to quantify the role of NBI fuelling vs turbulent pinch in determining density peaking at various collisionalities. Some attention starts to be given to the issue of the isotope effect, on which recent fresh data have become available.

Studies of pedestal stabilization away from the peeling -ballooning limit are focussing on the impact of impurities on microturbulence. Also studies of the dynamics of ELMs and their effects on pedestal and impurity accumulation were reported from DIII-D, EAST and KSTAR. The nonlinear findings are that zonal flows are playing an important role in pedestal collapse dynamics. WEST is starting operations in 2017 and offers new opportunities for transport studies in full $\mathrm{W}$ environment, with low torque and dominant electron RF heating.

\section{Acknowledgments}

On behalf of all the participants to the workshop, the authors would like to thank the Local Organizing Committee, P.Ricci (Chair), B.Labit, I.Martin, H.Varrin (Secretary), as well as the local host SPC, for the perfect organization of a very fruitful and interesting meeting. The EU TTF Chairs would like to acknowledge precious input from the US and Asian Pacific TTF Chairs in the definition of the scientific program. Part of the European work reported has been carried out within the framework of the EUROfusion Consortium and has received funding from the Euratom research and training programme 2014-2018 under grant agreement No 633053. The views and opinions expressed herein do not necessarily reflect those of the European Commission. 